\title{
Comparación de la calidad del coque siderúrgico obtenido a diferentes
}

\section{escalas}

\author{
J.L.G. Cimadevilla*, M.E. Díaz-Faes*, M.D. Casal*, C. Suárez*, C. Barriocanal*, \\ M.A. Díez* y R. Álvarez
}
Resumen La resistencia mecánica en frío del coque siderúrgico -evaluada mediante el ensayo IRSID-, la reactividad frente al $\mathrm{CO}_{2}$ (CRI) y la resistencia mecánica post-reacción (CSR) son los parámetros más importantes empleados para controlar la calidad del coque en España. En el INCAR, antes del cierre de la Coquería Experimental en Julio de 1999, se ha realizado un estudio para determinar la correlación existente entre la calidad del coque obtenido a escala semi-industrial -horno de $6 \mathrm{t}-\mathrm{y}$ a escala piloto -horno de pared móvil (HPM) de $250 \mathrm{~kg}$-. En este trabajo se describen los estudios realizados y los resultados obtenidos, los cuales permiten predecir la calidad del coque que se vaya a obtener a escala industrial a partir de los datos obtenidos en el HPM.

Palabras clave Calidad del coque de horno alto. Horno de pared móvil. Densidad de carga. Escala de carbonización.

\section{Coke quality comparison from samples obtained at different scales}

\begin{abstract}
Coke cold mechanical strength measured by means of the IRSID test, reactivity towards $\mathrm{CO}_{2}$ (CRI) and post-reaction strength (CSR) are the most important parameters for controlling coke quality in Spain. INCAR was running a semi-industrial coking test plant until July of 1999. Previous to this closure, a comparison of the coke quality was made between the $6 \mathrm{t}$ oven of this test plant and a $250 \mathrm{~kg}$ movable wall oven (MWO). It is thus possible to use the coke quality data obtained in the $250 \mathrm{~kg} \mathrm{MWO}$ to predict coke quality at semi-industrial and industrial scales.
\end{abstract}

Keywords Blast furnace coke quality. Movable wall oven. Bulk density. Carbonization scale.

\section{INTRODUCCIÓN}

Entre 1969 y 1999 el Instituto Nacional del Carbón (INCAR) ha estado colaborando con la industria siderúrgica española (Ensidesa/CSI/Aceralia) en el control de los carbones y de las mezclas utilizadas en sus coquerías industriales. Para este fin, se utilizaba la Coquería Experimental del IN. $\mathrm{CAR}^{[1]}$, una instalación única en su género que permitía obtener resultados directamente trasladables a la práctica industrial, con pequeñas correcciones, para así poder predecir la calidad del coque obtenido en las plantas industriales.
La Coquería Experimental del INCAR se cerró en el verano de 1999. Durante los últimos años de vida de la misma el INCAR, con la colaboración de Ensidesa, adquirió un horno de coquización de pared móvil (HPM) de $250 \mathrm{~kg}$ de capacidad, que permite medir la presión/empuje desarrollado por los carbones durante el proceso de coquización. Este horno también se puede utilizar para controlar la calidad del coque que se va a utilizar en el horno alto con inyección de carbón ${ }^{[2]}$.

En el presente trabajo se ofrecen los resultados obtenidos referentes a la comparación de la calidad del coque procedente del HPM de $250 \mathrm{~kg}$ con la de 
los producidos en el antiguo horno de $6 \mathrm{t}$ de la Coquería Experimental del INCAR.

\section{MATERIALES Y PROCEDIMIENTO EXPERIMEN- TAL}

\subsection{Horno de $6 \mathrm{t}$ de la Coquería Experimental}

La Coquería del INCAR ha sido previamente des-

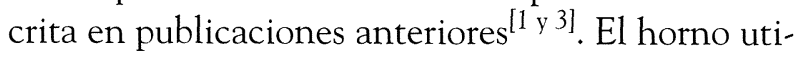
lizado ha sido el de 6 t de capacidad y la temperatura y el tiempo de coquización coinciden con los valores empleados en la práctica industrial.

\subsection{Horno de pared móvil de $\mathbf{2 5 0} \mathrm{kg}$ de capaci- dad}

Las principales características de este horno son las siguientes: $915 \times 840 \times 450 \mathrm{~mm}$ (longitud-altura-anchura); $250 \mathrm{~kg}$ de capacidad; temperaturas inicial y final de $880^{\circ} \mathrm{C}$ y $1.200^{\circ} \mathrm{C}$, respectivamente; $14^{\circ} \mathrm{C} / \mathrm{h}$ de velocidad de calentamiento y carga por gravedad.

Se trata de un horno eléctrico en el que el programa de calentamiento se fija para obtener un tiempo de coquización de $18 \mathrm{~h}$. Durante el ensayo se pueden medir la presión interna desarrollada, las temperaturas en el centro de la carga y en la pared y la contracción vertical. Los datos se registran y se muestran continuamente en un ordenador personal.

\subsection{Caracterización de la calidad de los coques obtenidos}

Aunque el INCAR ha desarrollado un método a escala de laboratorio para determinar la resistencia mecánica en frío de los coques ${ }^{[4]}$, en este trabajo, la calidad de los coques se determinó mediante dos tipos de ensayos habitualmente empleados por la industria siderúrgica española: la resistencia mecánica en frío determinada según el ensayo IRSID ${ }^{[5]}$ y la reactividad frente al $\mathrm{CO}_{2}$, determinada de acuerdo con el método NSC normalizado por ASTM, el cual permite obtener los índices CRI y CSR ${ }^{[6]}$ (resistencia mecánica post-reacción), y también mediante el método CEE-INCAR ${ }^{[7]}$ para determinación de la reactividad.

\section{RESULTADOS EXPERIMENTALES}

\subsection{Resistencia mecánica en frío: Ensayo IRSID}

Los ensayos realizados en los hornos de pared móvil son los que proporcionan a los operadores de las plantas industriales la seguridad de que una mezcla de carbones no va a desarrollar presiones peligrosas para la integridad de los hornos durante el proceso de coquización.

Asimismo, también se utilizan para determinar la calidad del coque. Para esta función, obviamente, es necesario que se pueda garantizar la transferencia de los resultados de escala piloto a escala industrial. En las figuras 1 y 2 se muestra la evolución de los valores del índice IRSID 20 (I20) de

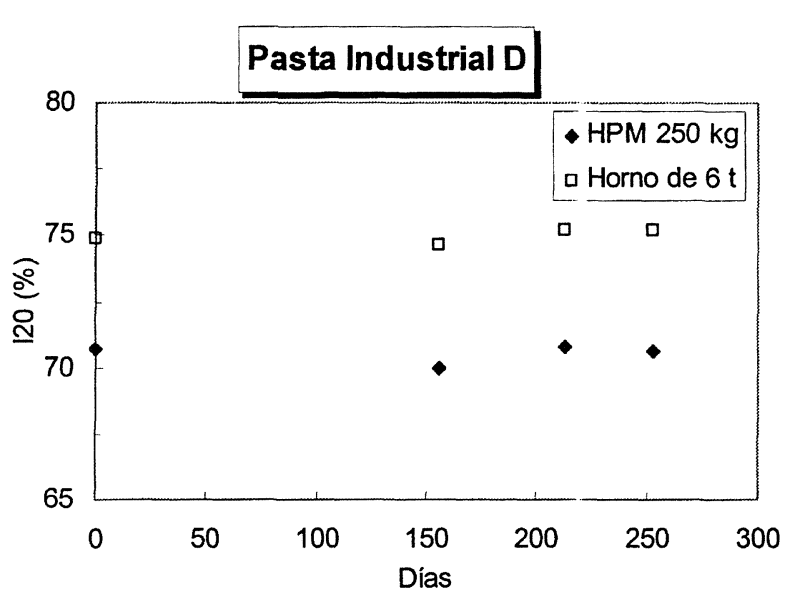

Figura 1. Comparación del índice 120 de coques obtenidos en el HPM de $250 \mathrm{~kg}$ y en el horno de 6 t en función del weathering de una pasta industrial.

Figure 1. Comparison of index 120 of cokes obtained at 250 $\mathrm{kg}$ MWO and at 6 toven with weathering of a industrial blend.

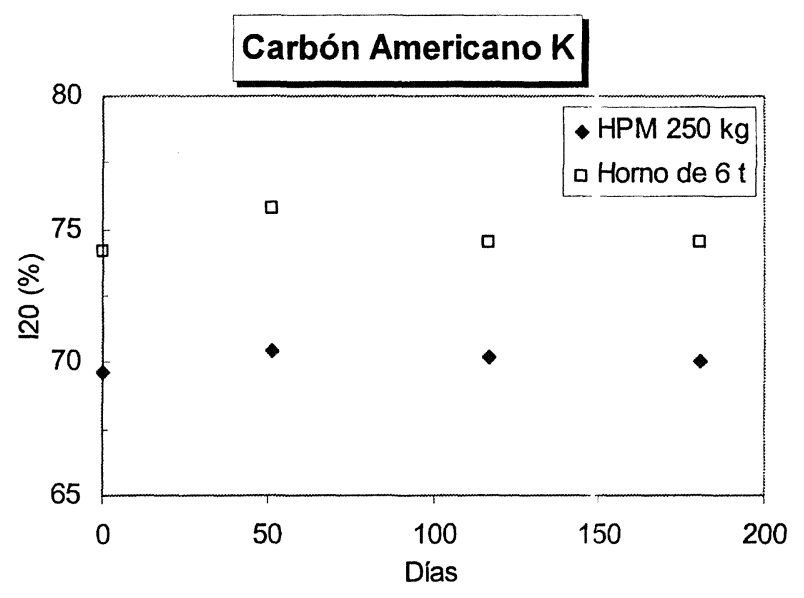

Figura 2. Comparación del índice 120 de coques obtenidos en el HPM de $250 \mathrm{~kg}$ y en el horno de 6 t en función del weathering del carbón americano $\mathrm{K}$.

Figure 2. Comparison of index 120 of cokes obtained at 250 $\mathrm{kg} \mathrm{MWO}$ and at 6 t oven with weathering of the american coal $K$.

Rev. Metal. Madrid Vol. Extr. (2005) 35-39 
coques obtenidos en el HPM de $250 \mathrm{~kg}$ y en el de $6 \mathrm{t}$, a partir de la pasta industrial $\mathrm{D}$ y de un carbón americano K obtenidos en un estudio de alteración meteórica de estos carbones ${ }^{[8]}$.

Se considera que para que un coque tenga un valor aceptable de I20 a escala industrial el valor en el horno de $6 \mathrm{t}$ del INCAR debería ser mayor de 74. En estas figuras se puede apreciar como, comparando el I20 del coque producido en ambos hornos para el carbón individual y para la mezcla en las mismas condiciones, el valor de I20 de los coques obtenidos en el HPM es considerablemente peor que el de los producidos en el horno de $6 \mathrm{t}$. Una posible explicación es que el coque del HPM tiene una menor cohesión debido a los efectos del tamaño de la cámara, efecto puertas y también al menor peso relativo de la carga en los hornos pequeños respecto a los hornos industriales. Otros autores han llegado a similares conclusiones ${ }^{[9}$ y 10$]$ en lo que respecta a la resistencia mecánica en frío de los coques.

Para obtener en el HPM valores del I20 semejantes a los del horno semi-industrial es necesario aumentar la densidad de carga respecto a la del horno de $6 \mathrm{t}$. En este último sentido, en la figura 3 se puede apreciar la sensible variación del I20 de los coques obtenidos en el HPM con la densidad de carga y también está incluido el valor del mismo a escala semi-industrial. Si se considera que el I20 aumenta linealmente con la densidad de carga, parece evidente que es necesario aumentar considerablemente la densidad de carga en el HPM respecto del horno semi-industrial para obtener valores similares del I20. En el caso del INCAR, se han fijado unos valores de densidad de carga para el HPM por encima de $750 \mathrm{~kg} / \mathrm{m}^{3}$, generalmente en el intervalo $750-820 \mathrm{~kg} / \mathrm{m}^{3}$ en base seca.

\subsection{Reactividad frente al $\mathrm{CO}_{2}$ (CRI y CEE-INCAR) y resistencia mecánica post-reacción (CSR)}

Respecto a la influencia de la densidad de carga sobre los índices CRI/CSR, en la figura 4 se puede ver un ejemplo del comportamiento de ambos parámetros para coques obtenidos en el HPM. El CRI no parece verse afectado, pero sí se aprecia con claridad una notable influencia sobre el CSR, el cual mejora sensiblemente con el aumento de la densidad de carga.

Hace ya bastante tiempo que se conocen las buenas correlaciones existentes entre el CRI y el
CSR y, desde fechas más recientes, entre el CRI y la reactividad CEE-INCAR ${ }^{[7 \text { y } 11]}$. En este último sentido, a modo de ejemplo, en la figura 5 se presenta la correlación que se ha encontrado entre ambas reactividades para coques producidos en el HPM. Como el ensayo de reactividad CEE-INCAR es más simple y mucho menos costoso que el de determinación de los parámetros CRI y CSR, podría llegar a sustituir a éste último y, además, abre la posibilidad de trabajar con menor cantidad de muestra de coque y de menor tamaño $(7 \mathrm{~g}$ de muestra de entre 1 y $3 \mathrm{~mm}$, frente a $200 \mathrm{~g}$ de coque con un tamaño entre 19 y $22,4 \mathrm{~mm}$, en el ensayo CRI/CSR).

\subsection{Comparación entre los coques obtenidos a diferentes escalas}

Finalmente, teniendo en cuenta lo anteriormente expuesto, en las figuras 6 y 7 se han representado, respectivamente, las correlaciones obtenidas para el I20 y para el CRI en el HPM y en el horno de $6 \mathrm{t}$.

A la vista de estas correlaciones, se deduce que se puede predecir la calidad de un coque que se vaya a obtener a escala industrial a partir de los datos obtenidos en el HPM.

\section{CONCLUSIONES}

En España, los parámetros más importantes para controlar la calidad del coque son la resistencia mecánica en frío, la reactividad frente al $\mathrm{CO}_{2}$ y la resistencia mecánica post-reacción. Los resultados mostrados en el presente trabajo, ponen de manifiesto que, empleando las condiciones de coquización adecuadas, es posible predecir la calidad de un coque siderúrgico que se vaya a producir a escala industrial a partir de los datos procedentes de coques obtenidos a escala piloto en un horno de pared móvil de $250 \mathrm{~kg}$. Asimismo, se ha encontrado una buena correlación entre los índices de reactividad frente al $\mathrm{CO}_{2}$, CRI y CEE-INCAR, para los coques obtenidos en el horno de pared móvil. Este último ensayo emplea una cantidad de muestra y un tiempo de reacción sensiblemente inferiores frente al ensayo CRI, de forma que se puede simplificar notablemente la obtención del dato de reactividad de las muestras de coque, al tiempo que se reduciría el coste de realización de dichos ensayos de reactividad. 

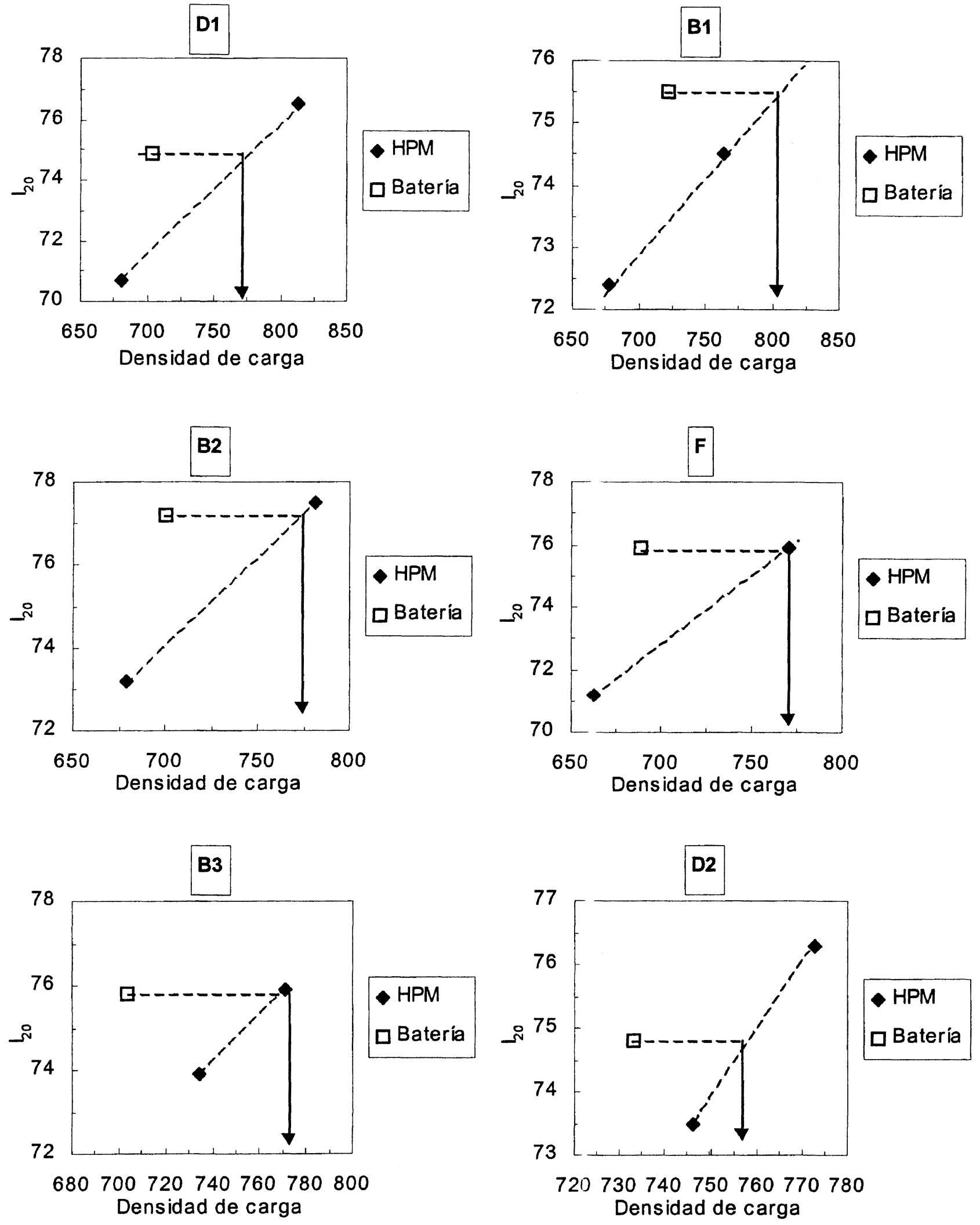

Figura 3. Variación del 120 con la densidad de carga.

Figure 3. Variation of index 120 with the bulk density. 


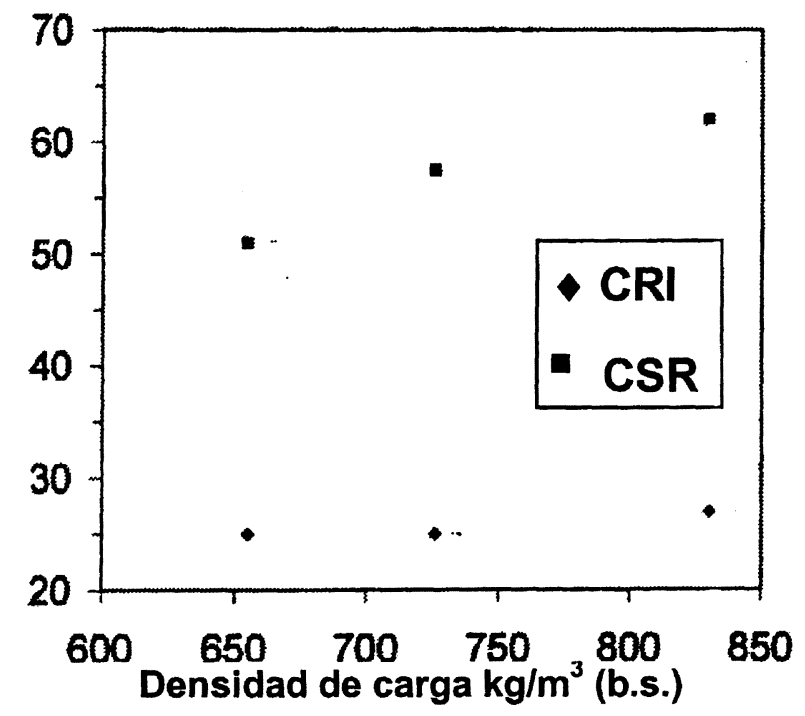

Figura 4. Cambios en los valores de los índices CRI/CSR con las variaciones de la densidad de carga (HPM $250 \mathrm{~kg}$ ).

Figure 4. Changes in CRI/CSR values with bulk density modifications (250 kg MWO).

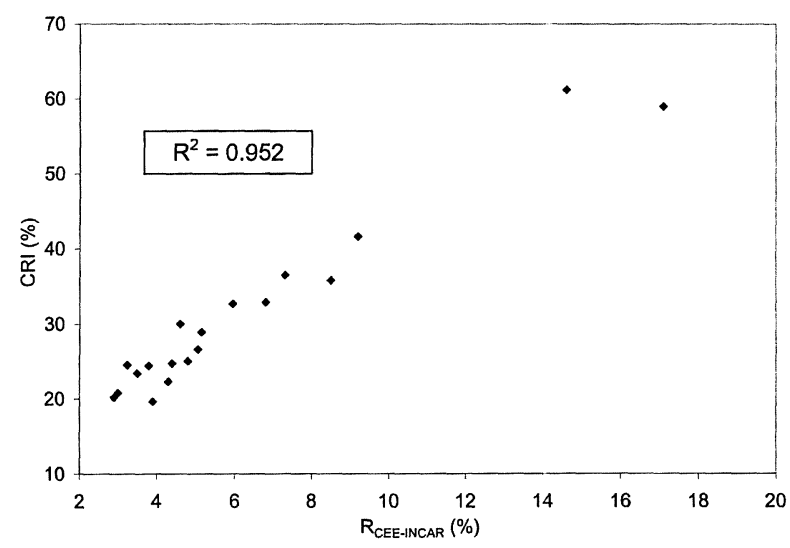

Figura 5. Comparación entre las reactividades CRI y CEEINCAR en el HPM de $250 \mathrm{~kg}$.

Figure 5. Comparison between CRI and CEE-INCAR reactivities in the $250 \mathrm{~kg} \mathrm{MWO}$.

\section{REFERENCIAS}

[1] R. Álvarez, E. Álvarez, C. SuÁrez, F. MÉndez de Andés y M. Sirgado, $1^{\text {st }}$ Int. Cokemaking Congress, Essen, Alemania, 1987, Brinck\&Co KG, E5, 20 p.

[2] H.W. Gudenau, D. Senk, K. Fukada, A. Babich, C. Froelhling, L.L. García, A. Formoso, FJ. Alguacil y A. Cores. Rev. Metal. Madrid 39 (2003) 367-377.

[3] M.A. Díez, R. Álvarez, M. Sirgado y H. Marsh, ISIJ Int. 31 (1991) 449-457.

[4] M.A. Díez, R. Álvarez, N. Radoslavov, C. Barriocanal, C.S. Canga y J. Xiberta, Rev. Metal. Madrid 38 (2002) 410-418.

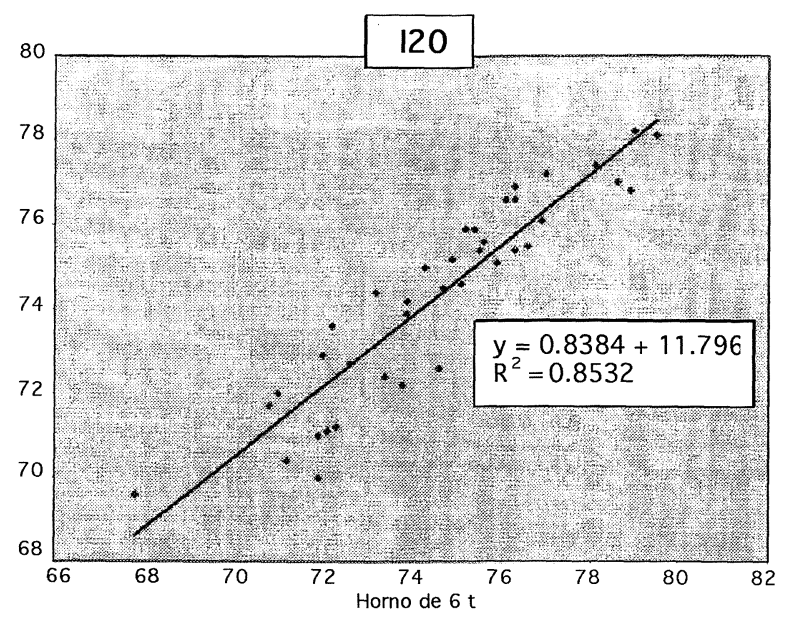

Figura 6. Correlación para el 120 de coques obtenidos en el HPM de $250 \mathrm{~kg}$ y en el horno de $6 \mathrm{t}$.

Figure 6. Comparison between index 120 of cokes obtained from $250 \mathrm{~kg} \mathrm{MWO}$ and 6 t oven.

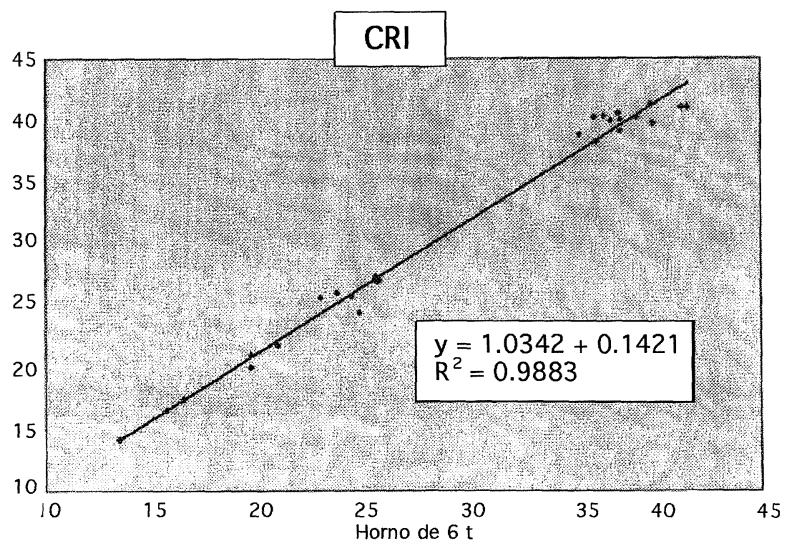

Figura 7. Correlación para el CRI de coques obtenidos en el HPM de $250 \mathrm{~kg}$ y en el horno de $6 \mathrm{t}$.

Figure 7. Comparison between index CRI of cokes obtained from $250 \mathrm{~kg}$ MWO and 6 t oven.

[5] UNE 32018, Coque. Determinación de la resistencia mecánica. Índices MICUM e IRSID (1982).

[6] ASTM D 5341-93, Standard test method for measuring Coke Reactivity Index (CRI) and Coke Strength after Reaction (CSR).

[7] J.A. Menéndez, R. Álvarez y J.J. Pis, Rev. Metal. Madrid 29 (1993) 214-222.

[8] R. Álvarez, C. Barriocanal, M.D. Casal, M.A. Díez, A.I. GONZÁlez, J.J. PIS y C. SUÁREZ, Proc. $55^{\text {th }}$ Ironmaking Conf., IIS-AIME, 1996, pp. 265-274.

[9] J.F. Gransden, M.A. Khan y J.T. Price, Proc. $44^{\text {th }}$ Ironmaking Conf., IIS-AIME, 1985, pp. 305-312.

[10] P. Arendt, F. Huhn, J. Strunk, U. Janhsen, J. Fohl, L. PERSSON y R. LinderT, Cokemak. Int. 101 (1998) 41-47.

[11] J.A. Menéndez, R. Álvarez y J.J. Pis, Ironmak. Steelmak. 26 (1999) 117-121. 